\title{
Some families of non-congruent numbers
}

\author{
by \\ Franz Lemmermeyer (San Marcos, CA)
}

1. Introduction. The elliptic curves $E_{k}: y^{2}=x\left(x^{2}-k^{2}\right)$ with $k \in \mathbb{Z}$ have been studied extensively, mainly because of their connection with the ancient problem of congruent numbers (see Guy [13] or Koblitz [17]). Many authors constructed families of non-congruent numbers by minimizing the Selmer groups attached to 2-isogenies of $E_{k}$ (see Feng [9, 10], Goto [12], Iskra [15], T. Ono [31], Serf [36], to name but the most recent contributors; actually results of this type go back to Genocchi [11] in the 19th century). Sharper results were obtained notably by J. Lagrange $[19,20]$ and, more recently, Wada [39], Nemenzo [28], and Li \& Tian [24], who found better bounds on the rank of $E_{k}$ by taking the 2-part of the Tate-Shafarevich groups into account. In this article, we will refine the criteria obtained by Lagrange and show that curves $E_{k}$, where $k=p l$ for primes $p \equiv l \equiv 1 \bmod 8$, very rarely have Tate-Shafarevich groups with trivial 2-part.

Notation. We recall the relevant notation from [22] (the standard reference for notions not explained here is Silverman [37]): elliptic curves $E$ with a rational point $T$ of order 2 as our curves $E_{k}$ come attached with a 2-isogeny $\phi: E \rightarrow \widehat{E}$ (depending on the choice of $T$ if $E$ has three rational points of order 2$)$. For $T=(0,0)$ we find the isogenous curve

$$
\widehat{E}_{k}: \quad y^{2}= \begin{cases}x\left(x^{2}+4 k^{2}\right) & \text { if } k \text { is odd } \\ x\left(x^{2}+k^{2} / 4\right) & \text { if } k \text { is even }\end{cases}
$$

(the distinction is made in order to minimize the coefficients of the curve; we could just as well work with only $y^{2}=x\left(x^{2}+4 k^{2}\right)$ as both models are isomorphic). The dual isogeny $\widehat{E}_{k} \rightarrow E_{k}$ will be denoted by $\psi$. If $k$ is fixed, we will suppress this index and write $E$ and $\widehat{E}$ for $E_{k}$ and $\widehat{E}_{k}$.

2000 Mathematics Subject Classification: Primary 11G05.

The main part of this article was written in 1999 while the author was at the MPI Bonn; he would like to thank everyone there for the hospitality and stimulating environment, and the DFG for financial support during that time. 
Consider the torsors (often also called principal homogeneous spaces)

$$
\begin{aligned}
& \mathcal{T}^{(\psi)}\left(b_{1}\right): \quad N^{2}=b_{1} M^{4}+b_{2} e^{4}, \quad b_{1} b_{2}=-k^{2}, \\
& \mathcal{T}^{(\phi)}\left(b_{1}\right): \quad N^{2}=b_{1} M^{4}+b_{2} e^{4}, \quad b_{1} b_{2}= \begin{cases}4 k^{2} & \text { if } k \text { is odd }, \\
k^{2} / 4 & \text { if } k \text { is even. }\end{cases}
\end{aligned}
$$

The Selmer group $\operatorname{Sel}^{(\psi)}(\widehat{E} / \mathbb{Q})$ is defined as the subgroup of $\mathbb{Q}^{\times} / \mathbb{Q}^{\times 2}$ consisting of classes $b_{1} \mathbb{Q}^{\times 2}$ such that $\mathcal{T}^{(\psi)}\left(b_{1}\right)$ has a non-trivial $(\neq(0,0,0))$ rational point in every completion $\mathbb{Q}_{v}$ of $\mathbb{Q}$; the subgroup of $\operatorname{Sel}^{(\psi)}(\widehat{E} / \mathbb{Q})$ such that the torsors $\mathcal{T}^{(\psi)}\left(b_{1}\right)$ corresponding to $b_{1} \mathbb{Q}^{\times 2}$ have a rational point will be denoted by $W(\widehat{E} / \mathbb{Q}$ ) (from now on, rational point will stand for non-trivial rational point; we may and do assume moreover that its coordinates are integral and primitive, that is, $(M, e)=1)$. Similarly we define $\operatorname{Sel}^{(\phi)}(E / \mathbb{Q})$ and $W(E / \mathbb{Q})$. Finally, the Tate-Shafarevich groups are defined via the exact sequences

$$
\begin{aligned}
& 0 \rightarrow W(E / \mathbb{Q}) \rightarrow \operatorname{Sel}^{(\phi)}(E / \mathbb{Q}) \rightarrow \amalg(E / \mathbb{Q})[\phi] \rightarrow 0, \\
& 0 \rightarrow W(\widehat{E} / \mathbb{Q}) \rightarrow \operatorname{Sel}^{(\psi)}(\widehat{E} / \mathbb{Q}) \rightarrow \amalg(\widehat{E} / \mathbb{Q})[\psi] \rightarrow 0 .
\end{aligned}
$$

Below, we will often write $\langle x, \ldots, z\rangle$ for the subgroup $\left\langle x \cdot \mathbb{Q}^{\times 2}, \ldots, z \cdot \mathbb{Q}^{\times 2}\right\rangle$ of $\mathbb{Q}^{\times} / \mathbb{Q}^{\times 2}$ generated by $x, \ldots, z$.

The Selmer and Tate-Shafarevich groups attached to a pair of isogenies $\phi$ and $\psi$ with $\psi \circ \phi=[n]$ for some integer $n \geq 2$ are related to the $n$-torsion of Selmer and Tate-Shafarevich groups as follows (see diagram (3.9) in Razar $[32$, p. 139]; Feng $[9,10]$ erroneously claims that we always have $C=\widehat{C}=0)$ :

Proposition 1. With the notation as above, we have the following exact and commutative diagram:

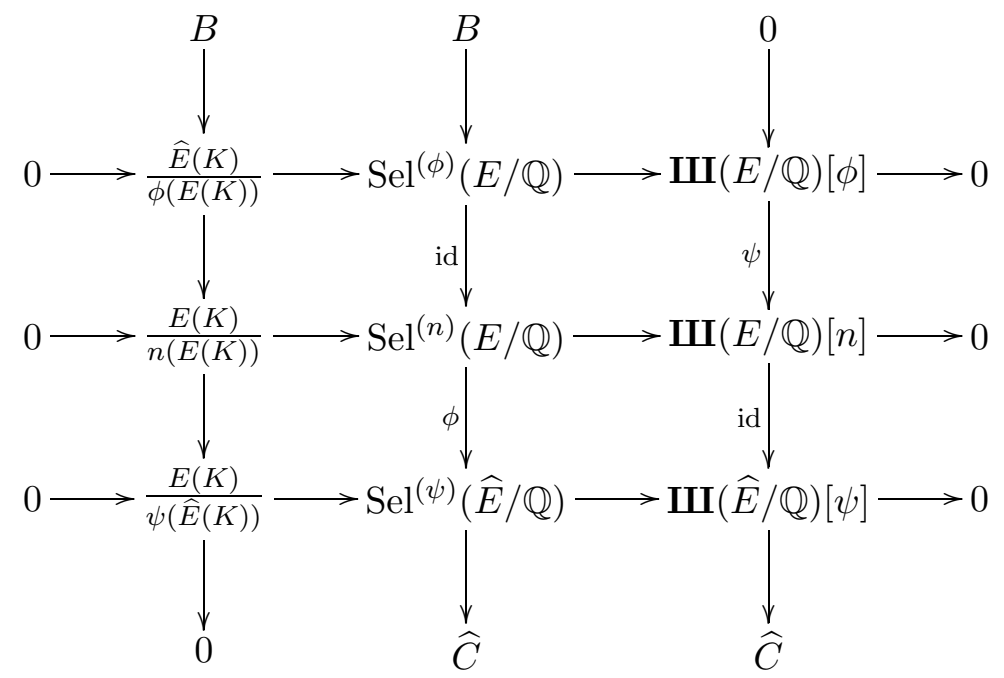


Here, the vertical maps from $B$ are injections, and those into $\widehat{C}$ are surjections. There is a corresponding diagram with the roles of $\phi$ and $\psi$ reversed, and with groups $\widehat{B}$ and $C$. Moreover, $C$ and $\widehat{C}$ are groups of even rank.

There exist various methods for constructing elements of order 2 in Tate-Shafarevich groups: one can perform a second 2-descent (cf. Birch and Swinnerton-Dyer [2], Razar [32], Lagrange [19, 20], Wada [39] and Nemenzo [27]), employ the Cassels pairing (see e.g. Aoki [1], Bölling [3], Cassels [5], and McGuinness [26]), compare the Selmer groups $\operatorname{Sel}^{(\psi)}(\widehat{E} / \mathbb{Q})$ and $\operatorname{Sel}^{(2)}(E / \mathbb{Q})$ as done by Kramer [18] (essentially, the methods mentioned so far are all equivalent to the classical second 2-descent), or use the method usually attributed to Lind [25] but actually going back (in a slightly different context) to Rédei [33] and Dirichlet [8] (I learned this technique from Stroeker \& Top [38] and used it in [22] and [21]). In this paper, we continue to use this last method; as we shall see, it will allow us to obtain results that are stronger than those provided by simple second 2-descents.

Our main results are the solvability criteria in Table 4 below; this will imply the lower bound $1 / 2$ for the density of rank-0 curves among the $E_{p l}$.

2. Preliminaries. In the calculations below we will have use quite a number of elementary results on quadratic reciprocity and genus theory. The following subsections recall what we will need.

2.1. Some reciprocity laws. In the following, $p$ and $l$ will denote primes $\equiv 1 \bmod 8$, and $\pi$ and $\lambda$ will denote primary primes in $\mathbb{Z}[i]$ with norms $p$ and $l$, respectively. A prime $\pi$ of norm $p \equiv 1 \bmod 8$ is called primary if $\pi$ is congruent to a square modulo 4 . For $\pi \in \mathbb{Z}[i], \Pi \in \mathbb{Z}[\sqrt{2}]$ and $\Pi^{*} \in \mathbb{Z}[\sqrt{-2}]$ we can always choose associates satisfying $\pi \equiv 1 \bmod 2+2 i, \Pi \equiv 1 \bmod 2 \sqrt{2}$ and $\Pi^{*} \equiv 1 \bmod 2 \sqrt{-2}$, and these elements are primary.

We will need a few elementary results on quadratic residue symbols; as in [22], we let $(p / l)_{4}$ denote the biquadratic residue symbol for primes $l \equiv 1 \bmod 4$ such that $(p / l)=1$, and we let $[\cdot / \cdot]$ denote the quadratic residue symbol in $\mathbb{Z}[i]$. We also note that for primes $l=\lambda \bar{\lambda} \equiv 1 \bmod 8$, the relation $(1+i)^{4}=-4$ implies that $[1+i / \lambda]=(-4 / l)_{8}$ (this is the rational octic residue symbol). Moreover, $[\pi / \lambda]=(p / l)_{4}(l / p)_{4}$ for primes $p=\pi \bar{\pi}$ and $l=\lambda \bar{\lambda}$ such that $(p / l)=1$ by Burde's rational reciprocity law. Finally, it is easy to check that $\left(\varepsilon_{2} / p\right)=[1+i / \pi]=(-4 / p)_{8}$, where $\varepsilon_{2}=1+\sqrt{2}$ (see $[23])$.

Now recall that primes $p \equiv 1 \bmod 8$ are norms from $\mathbb{Z}\left[\zeta_{8}\right]$, say $p=N \alpha$ for some $\alpha \equiv 1 \bmod \left(2+2 \zeta_{8}\right)$, and in fact there exist primary elements $\pi \in \mathbb{Z}[i]$, $\Pi \in \mathbb{Z}[\sqrt{2}]$ and $\Pi^{*} \in \mathbb{Z}[\sqrt{-2}]$ with norm $p$. For primes $l \equiv 1 \bmod 8$, we define $\lambda, \Lambda$ and $\Lambda^{*}$ similarly. Unless explicitly stated otherwise, this notation is valid for the rest of this article. 
The following result shows that solvability criteria involving the quadratic symbol $\left[\Pi^{*} / \Lambda^{*}\right]$ can be reduced to criteria involving only $[\Pi / \Lambda]$ and rational quartic residue symbols:

Proposition 2. Let $p \equiv l \equiv 1 \bmod 8$ be primes such that $(p / l)=+1$. Then

$$
\left[\frac{\Pi}{\Lambda}\right]\left[\frac{\Pi^{*}}{\Lambda^{*}}\right]=\left[\frac{\pi}{\lambda}\right]=\left(\frac{p}{l}\right)_{4}\left(\frac{l}{p}\right)_{4},
$$

where the first three symbols $[\cdot / \cdot]$ denote the quadratic residue symbol in $\mathbb{Z}[\sqrt{2}], \mathbb{Z}[\sqrt{-2}]$ and $\mathbb{Z}[i]$, respectively.

Proof. We know that there exists an element $\alpha \in \mathbb{Z}\left[\zeta_{8}\right]$ such that $\Pi^{*}=$ $\alpha_{1} \alpha_{3}$ (here $\alpha_{j}=\sigma_{j}(\alpha)$, where $\sigma_{j}$ is the automorphism that sends $\zeta_{8}$ to $\zeta_{8}^{j}$; in particular $\alpha_{1}=\alpha$ ), $\pi=\alpha_{1} \alpha_{5}$ and $\Pi=\alpha_{1} \alpha_{7}$ (observe that such norms are necessarily totally positive). Defining $\beta$ accordingly we have $[\Pi / \Lambda]=$ $\left(\alpha_{1} \alpha_{7} / \beta\right)$, where $(\cdot / \cdot)$ is the quadratic residue symbol in $\mathbb{Z}\left[\zeta_{8}\right]$. Similarly, we have $\left[\Pi^{*} / \Lambda^{*}\right]=\left(\alpha_{1} \alpha_{3} / \beta\right)$, hence $[\Pi / \Lambda]\left[\Pi^{*} / \Lambda^{*}\right]=\left(\alpha_{3} \alpha_{7} / \beta\right)$. But this last symbol equals $[\bar{\pi} / \lambda]$, and since $(p / l)=+1$ this coincides with $[\pi / \lambda]$. This proves our claim by Burde's reciprocity law.

We also note that $[\Lambda / \Pi]=[\Pi / \Lambda]$ and $\left[\Lambda^{*} / \Pi^{*}\right]=\left[\Pi^{*} / \Lambda^{*}\right]$ by the quadratic reciprocity laws in $\mathbb{Z}[\sqrt{2}]$ and $\mathbb{Z}[\sqrt{-2}]$, respectively. Finally, if $K / k$ is an extension of number fields, if $[\cdot / \cdot]$ and $(\cdot / \cdot)$ denote the quadratic residue symbols in $K$ and $k$, respectively, and if $\mathfrak{a}$ is an ideal in $\mathcal{O}_{k}$ with odd norm, then $[\alpha / \mathfrak{a}]=\left(N_{K / k} \alpha / \mathfrak{a}\right)$ directly from the definition of residue symbols. Similarly, for ideals $\mathfrak{A}$ in $\mathcal{O}_{K}$ with relative norm $\mathfrak{a}$ and elements $\alpha \in k$ coprime to $\mathfrak{a}$, we have $[\alpha / \mathfrak{A}]=(\alpha / \mathfrak{a})$. For more on rational reciprocity laws, see [23, Chap. 5].

2.2. The class groups of $\mathbb{Q}(\sqrt{ \pm 2 l})$. Let us begin by reviewing the basic results of Scholz as pertaining to the special case $k=\mathbb{Q}(\sqrt{2 l})$, where $l \equiv$ $1 \bmod 4$ is prime. Let $\varepsilon, h$ and $h^{+}$denote the fundamental unit, the class number and the class number in the strict sense of $k$. Moreover, define $(l / 2)_{4}=(-1 / l)_{8}$ for primes $l \equiv 1 \bmod 8$; then $(-4 / l)_{8}=(2 / l)_{4}(l / 2)_{4}$. The following proposition is the special case $p=2$ of a more general result due to Scholz [34]:

Proposition 3. With the notation as above, there are the following cases:

- $(2 / l)=-1:$ then $N \varepsilon=-1$ and $h \equiv h^{+} \equiv 2 \bmod 4$;

- $(2 / l)=+1$ :

(1) if $(2 / l)_{4}=-(l / 2)_{4}$, then $N \varepsilon=+1, h \equiv 2 \bmod 4$, and $h^{+} \equiv$ $4 \bmod 8$

(2) if $(2 / l)_{4}=(l / 2)_{4}=-1$, then $N \varepsilon=-1$ and $h \equiv h^{+} \equiv 4 \bmod 8$; 
(3) if $(2 / l)_{4}=(l / 2)_{4}=+1$, then $4 \mid h$ and $8 \mid h^{+}$.

Note that the prime ideal 2 above 2 in $\mathbb{Q}(\sqrt{2 l})$ is principal in the usual sense if and only if $N \varepsilon=+1$ for the fundamental unit $\varepsilon$ of $\mathbb{Q}(\sqrt{2 l})$. This follows by applying the class number formula for strictly ambiguous ideals $C_{\text {am }}=2^{t-1} /\left(E_{F} / N E_{K}\right)$ in quadratic extensions $K / F$, where $t$ denotes the number of ramified primes, and where $E_{F}$ and $E_{K}$ are the unit groups of $\mathcal{O}_{F}$ and $\mathcal{O}_{K}$, respectively.

Let $\mathfrak{a} \stackrel{+}{\sim} 2$ be short for "the ideal $\mathfrak{a}$ is equivalent in the strict sense to the square of some ideal", and define $\mathfrak{a} \stackrel{ \pm}{\sim} 4$ similarly.

If $d=d_{1} d_{2}$ is a product of two prime discriminants, then classical genus theory tells us that for some ideal $\mathfrak{a}$ with norm $a$ (the existence of $\mathfrak{a}$ implies $(d / a)=+1)$, we have $\mathfrak{a} \stackrel{ \pm}{\sim} 2$ if and only if $\left(d_{1} / a\right)=\left(d_{2} / a\right)=+1$.

Lemma 4 . Let $p \equiv l \equiv 1 \bmod 8$ be primes such that $(p / l)=+1$, and let $\mathfrak{p}$ denote the prime ideal above $p$ in $k=\mathbb{Q}(\sqrt{2 l})$. Then $\mathfrak{p} \stackrel{+}{\sim} 4 \Leftrightarrow[\Lambda / \Pi]=1$.

Proof. If $4 \mid h^{+}$, then the corresponding quartic cyclic unramified extension $K / k$ is given by $K=k(\sqrt{\Lambda})$. A prime ideal $\mathfrak{p}$ of degree 1 will split completely in $K / k$ if and only if its ideal class is a fourth power in $\mathrm{Cl}^{+}(k)$; on the other hand, Kummer theory shows that $\mathfrak{p}$ splits if and only if $\Lambda$ is a quadratic residue modulo any prime ideal above $\mathfrak{p}$ in $\mathbb{Q}(\sqrt{2})$, that is, if and only if $[\Lambda / \Pi]=1$.

Lemma 5. Let $k=\mathbb{Q}(\sqrt{2 l})$ and assume that $(-4 / l)_{8}=-1$. Then the prime ideal 2 above 2 in $\mathcal{O}_{k}$ is principal in the strict sense if and only if $(2 / l)_{4}=-1$.

Proof. First observe that our assumption implies by Proposition 3 that the fundamental unit of $k$ has positive norm, that 2 is principal in the wide sense, and that $h^{+} \equiv 4 \bmod 8$.

Assume that 2 is principal in the strict sense. Then $X^{2}-2 l y^{2}=+2$ is solvable, hence so is $2 x^{2}-l y^{2}=1$ (we have put $X=2 x$ ). Now clearly $2 \nmid x$, hence $x^{2} \equiv 1 \bmod 8$ and $2 x^{2} \equiv 2 \bmod 16$; on the other hand, $(2 / y)=+1$, hence $y^{2} \equiv 1 \bmod 16$. Together this implies that $l \equiv 1 \bmod 16$, that is, $(-1 / l)_{8}=+1$. Since $(-4 / l)_{8}=-1$ by assumption, this is equivalent to $(2 / l)_{4}=-1$.

Now assume that 2 is not principal in the strict sense. Then $X^{2}-2 l y^{2}=$ -2 , and with $X=2 x$ we get $2 x^{2}-l y^{2}=-1$. Now $(2 / l)_{4}=(x / l)=\left(l / x^{\prime}\right)$, where $x=2^{j} x^{\prime}$ with $x^{\prime}$ odd, and $\left(l / x^{\prime}\right)=+1$ by reducing our equation modulo $x^{\prime}$. Thus $(2 / l)_{4}=+1$.

3. The case $k=2 p$. We will now investigate which torsors of $E_{2 p}$ do not have rational points although they are everywhere locally solvable. These curves were already studied by Lagrange [20] using second 2-descents and 
by Kings $[16]$ using the Cassels pairing on $\amalg(E / \mathbb{Q})$. The curves $E_{2 p}$ are the simplest examples where $\amalg(E / \mathbb{Q})[\phi]$ and $\amalg(\widehat{E} / \mathbb{Q})[\psi]$ may have odd dimension:

THEOREM 6 . Let $p \equiv 1 \bmod 8$ be a prime and consider the elliptic curve $E: y^{2}=x\left(x^{2}-4 p^{2}\right)$. Then the Selmer groups are given by

$$
\operatorname{Sel}^{(\psi)}(\widehat{E} / \mathbb{Q})=\langle-1,2, p\rangle, \quad \operatorname{Sel}^{(\phi)}(E / \mathbb{Q})=\langle p\rangle,
$$

and if $p \equiv 9 \bmod 16$, then $\amalg(\widehat{E} / \mathbb{Q})[\psi]=\langle p\rangle$ and $\amalg(E / \mathbb{Q})[\phi]=\langle p\rangle$. Moreover, $\mathbf{\amalg}(E / \mathbb{Q})[2] \simeq \mathbf{\amalg}(\widehat{E} / \mathbb{Q})[2] \simeq(\mathbb{Z} / 2 \mathbb{Z})^{2}$.

Proof. We leave the proofs that $\amalg(\widehat{E} / \mathbb{Q})[\psi]$ and $\amalg(E / \mathbb{Q})[\phi]$ both have order 2 as an exercise to the reader (they are much simpler than the proofs in the sections below). The claims $\amalg(E / \mathbb{Q})[2] \simeq \amalg(\widehat{E} / \mathbb{Q})[2] \simeq(\mathbb{Z} / 2 \mathbb{Z})^{2}$ follow from the exact sequences extracted from the diagram in Proposition 1:

$$
\begin{aligned}
& 0 \rightarrow \amalg(\widehat{E} / \mathbb{Q})[\psi] \rightarrow \amalg(\widehat{E} / \mathbb{Q})[2] \rightarrow \amalg(E / \mathbb{Q})[\phi] \rightarrow C \rightarrow 0, \\
& 0 \rightarrow \amalg(E / \mathbb{Q})[\phi] \rightarrow \amalg(E / \mathbb{Q})[2] \rightarrow \amalg(\widehat{E} / \mathbb{Q})[\psi] \rightarrow \widehat{C} \rightarrow 0,
\end{aligned}
$$

where $C$ and $\widehat{C}$ are finite groups of square order by results of Cassels (this follows from the existence of the Cassels pairing on $\mathbf{\amalg}$, first proved in [6] in the special case of curves $x^{3}+y^{3}+d z^{3}=0$. The special case that we need here simply expresses the fact that the difference between the rank estimates of the first and second descent is always even). Since in our case they are quotients of groups of order 2 , it follows that $C=\widehat{C}=0$, and this implies our claim.

4. The case $k=p l \equiv 1 \bmod 8$. The simplest cases are those where $p$ and $l$ are primes such that $p \equiv l \equiv 3,5,7 \bmod 8$; they were already discussed by Lagrange [19]; Table 1 gives the Selmer groups $\operatorname{Sel}^{(\psi)}(\widehat{E} / \mathbb{Q})$ and $\operatorname{Sel}^{(\phi)}(E / \mathbb{Q})$ attached to the 2-isogenies described above.

Table 1. Selmer groups $\operatorname{Sel}^{(\psi)}(\widehat{E} / \mathbb{Q})$ and $\operatorname{Sel}^{(\phi)}(E / \mathbb{Q})$ for $E$ and $\widehat{E}$, where $p$ and $l$ are primes such that $p l \equiv 1 \bmod 8$.

\begin{tabular}{ccccc}
\hline$p \bmod 8$ & $l \bmod 8$ & $(p / l)$ & $\operatorname{Sel}^{(\psi)}(\widehat{E} / \mathbb{Q})$ & $\operatorname{Sel}^{(\phi)}(E / \mathbb{Q})$ \\
\hline 1 & 1 & +1 & $\langle-1, p, l\rangle$ & $\langle 2, p, l\rangle$ \\
& & -1 & $\langle-1, p l\rangle$ & $\langle 2, p l\rangle$ \\
5 & 5 & +1 & $\langle-1, p l\rangle$ & $\langle p, l\rangle$ \\
& & -1 & $\langle-1, p l\rangle$ & $\langle 2 p, 2 l\rangle$ \\
3 & 3 & & $\langle-1, p l\rangle$ & 1 \\
7 & 7 & & $\langle-1, p, l\rangle$ & $\langle 2\rangle$ \\
\hline
\end{tabular}

Lagrange also found necessary criteria for the solvability of certain torsors. Here are the results, reformulated using our notation: 
Proposition 7. Let $p$ and $l$ be distinct primes such that $p \equiv l \equiv$ 3, 5, 7 mod 8. If the torsors in each row of the table below have a rational point, then the conditions in the last column of the corresponding row must be satisfied:

\begin{tabular}{ccccc}
\hline$p \bmod 8$ & $l \bmod 8$ & $(p / l)$ & Torsors & Conditions \\
\hline 5 & 5 & +1 & $\mathcal{T}^{(\phi)}(p), \mathcal{T}^{(\phi)}(l), \mathcal{T}^{(\phi)}(p l)$ & $(p / l)_{4}=(l / p)_{4}$ \\
5 & 5 & -1 & $\mathcal{T}^{(\phi)}(2 p), \mathcal{T}^{(\phi)}(2 l), \mathcal{T}^{(\phi)}(p l)$ & {$[(1+i) \pi / \lambda]=+1$} \\
7 & 7 & +1 & $\mathcal{T}^{(\phi)}(2), \mathcal{T}^{(\psi)}(p), \mathcal{T}^{(\psi)}(l)$ & {$[\Lambda / \Pi]=+1$} \\
\hline
\end{tabular}

In the last row, $\Lambda \in \mathbb{Z}[\sqrt{2}]$ is a primary element with norm -l, and $\Pi \in \mathbb{Z}[\sqrt{2}]$ has norm $\pm p$.

Observe that $[\Lambda / \Pi]$ is well defined since $[\Lambda / \Pi][\bar{\Lambda} / \Pi]=[-l / \Pi]=(-l / p)$ $=(p / l)=+1$.

The proofs for $p \equiv l \equiv 5 \bmod 8$ are straightforward and left as an exercise to the reader. Here we give some details for the case $p \equiv l \equiv 7 \bmod 8$. Consider the torsor $\mathcal{T}(p): p n^{2}=M^{4}-l^{2} e^{4}$. Reduction modulo $l$ shows immediately that either 1) $l \nmid M$ and $(p / l)=+1$, or 2$) l \mid M$ and $(p / l)=-1$. Moreover, either A) $2 \nmid M e$ and $2 \mid n$, or B) $2 \nmid n e$ and $2 \mid M$. As in the case $p \equiv l \equiv 1 \bmod 8$, we get four equations per case:

\begin{tabular}{lcccc}
\hline Case & I & II & III & IV \\
\hline $1 \mathrm{~A})$ & $M^{2}+l e^{2}=2 p a^{2}$ & $M^{2}-l e^{2}=2 b^{2}$ & $p a^{2}+b^{2}=M^{2}$ & $p a^{2}-b^{2}=l e^{2}$ \\
$1 \mathrm{~B})$ & $M^{2}+l e^{2}=p a^{2}$ & $M^{2}-l e^{2}=b^{2}$ & $p a^{2}+b^{2}=2 M^{2}$ & $p a^{2}-b^{2}=2 l e^{2}$ \\
$2 \mathrm{~A})$ & $l m^{2}+e^{2}=2 a^{2}$ & $l m^{2}-e^{2}=2 p b^{2}$ & $a^{2}-p b^{2}=e^{2}$ & $a^{2}-p b^{2}=l m^{2}$ \\
2B) & $l m^{2}+e^{2}=a^{2}$ & $l m^{2}-e^{2}=p b^{2}$ & $a^{2}-p b^{2}=2 e^{2}$ & $a^{2}-p b^{2}=2 l m^{2}$ \\
\hline
\end{tabular}

Now we consider these four cases separately:

1A) Writing II and III in the form $M^{2}-2 b^{2}=l e^{2}$ and $M^{2}-b^{2}=p a^{2}$ we find that $[\lambda / \Pi]=[M+b \sqrt{2} / \Pi]$, where $\lambda \in \mathbb{Z}[\sqrt{2}]$ is the element of norm $l$ that divides $M+b \sqrt{2}$. We would like to use the congruence $b \equiv \pm M \bmod p$ coming from the second equation and conclude that $[\lambda / \Pi]=[1 \pm \sqrt{2} / \Pi]$, but unfortunately the last symbol depends on the choice of sign. We therefore have to work a little harder.

First observe that for $M>0$, we have $(M / p)=(-p / M)=+1$ from the second equation. Now we factor $p a^{2}=(M-b)(M+b)$ and consider the following two cases:

a) $M-b=2 p r^{2}, M+b=2 s^{2}$ (the negative signs cannot hold here: otherwise we would get $M=-s^{2}-r p^{2}$, contradicting our assumption that $M>0)$; then $b=s^{2}-p r^{2} \equiv 1 \bmod 4$, and $[M+b \sqrt{2} / \Pi]=(M / p)[1+\sqrt{2} / \Pi]$ $=[1+\sqrt{2} / \Pi]$. 
b) $M-b=2 r^{2}, M+b=2 p s^{2}$; then $b=p s^{2}-r^{2} \equiv 3 \bmod 4$, and now $[M+b \sqrt{2} / \Pi]=[1-\sqrt{2} / \Pi]$.

Thus $[\lambda \varepsilon / \Pi]=+1$, where $\varepsilon=1+(-1 / b) \sqrt{2}$. Now it is easy to check that $\lambda \varepsilon$ is primary: in fact, $r+s \sqrt{2}$ with $2 \mid s$ is primary if and only if $r+s \equiv 1 \bmod 4$, and since $\lambda \varepsilon$ is primary if and only if $(M+b \sqrt{2}) \varepsilon$ is, we find

$$
(M+b \sqrt{2}) \varepsilon= \begin{cases}M+2 b+(M+b) \sqrt{2} & \text { if } b \equiv 1 \bmod 4, \\ M-2 b+(b-M) \sqrt{2} & \text { if } b \equiv 3 \bmod 4,\end{cases}
$$

and $2 M+3 b \equiv 2-b \equiv 1 \bmod 4$ in the first and $-b \equiv 1 \bmod 4$ in the second case. Thus in this case $[\Lambda / \Pi]=+1$, where $\Lambda=\lambda \varepsilon$ is primary with norm $-l$.

1B) Here $b^{2}-2 M^{2}=-p a^{2}$ and $b^{2}-M^{2}=-l e^{2}$. Again, choosing $M>0$ guarantees $(M / l)=(-l / M)=+1$. Next, $M-b=r^{2}$ and $M+b=l s^{2}$ imply $2 b=l s^{2}-r^{2}$ and $b \equiv 1 \bmod 4$, while $M-b=l r^{2}$ and $M+b=s^{2}$ give $2 b=s^{2}-l r^{2}$ and $b \equiv 3 \bmod 4$. Thus $[b+M \sqrt{2} / \Lambda]=[-1+\sqrt{2} / \Lambda]$ if $b \equiv 1 \bmod 4$, and $[b+M \sqrt{2} / \Lambda]=[1+\sqrt{2} / \Lambda]$ if $b \equiv 3 \bmod 4$. Putting $\varepsilon=$ $-(-1 / b)+\sqrt{2}$, it is easy to check that $(b+M \sqrt{2}) \varepsilon$ is totally positive. Now Hasse [14] has shown that we have the reciprocity law $[\alpha / \beta]=[\beta / \alpha]$ in an arbitrary algebraic number field if the conductors of $\alpha$ and $\beta$ are coprime. Since $(b+M \sqrt{2}) \varepsilon \gg 0$, the gcd of the conductors of $(b+M \sqrt{2}) \varepsilon$ and $\Lambda$ do not contain infinite primes, and since $\Lambda$ is primary, the gcd does not contain primes above 2 . But then $(b+M \sqrt{2}, \Lambda)=(1)$ guarantees that the conductors are indeed coprime, and the reciprocity law gives $1=[(b+M \sqrt{2}) \varepsilon / \Lambda]=$ $[\Lambda / b+M \sqrt{2}]$. Since $(b+M \sqrt{2})=\left(\Pi \alpha^{2}\right)$ by unique factorization, we conclude that $[\Lambda / b+M \sqrt{2}]=[\Lambda / \Pi]$.

2A) Equations I and III correspond to III and II in case 1B) with the roles of $p$ and $l$ switched.

2B) Again, this reduces to case 1A).

We have proved:

Proposition 8. Let $d_{i}$ denote the density of rank 0 curves among the $E_{p l}$, where $p \equiv l \equiv i \bmod 8$ are primes. Then $d_{3}=1, d_{5} \geq 1 / 2$ and $d_{7} \geq 1 / 2$.

The main result of this paper is that also $d_{1} \geq 1 / 2$ (this is much stronger than the result obtained by Lagrange [20]). Although numerical computations seem to suggest that $d_{i}=1$, it seems that the bounds derived in this article cannot be improved using our methods.

From now on, we will assume that $p$ and $l$ are both primes $\equiv 1 \bmod 8$.

4.1. The case $(p / l)=-1$. Let $k=p l$ be a product of primes $p \equiv l \equiv$ $1 \bmod 8$ with $(p / l)=-1$. Then $($ see $[20])$

$$
\operatorname{Sel}^{(\psi)}(\widehat{E} / \mathbb{Q})=\langle-1, p l\rangle=W(\widehat{E} / \mathbb{Q}), \quad \operatorname{Sel}^{(\phi)}(E / \mathbb{Q})=\langle 2, p l\rangle .
$$


In particular, $\amalg(\widehat{E} / \mathbb{Q})[\psi]=0$, so we only have to discuss the $\phi$-part of $\amalg(E / \mathbb{Q})$. Note that $\amalg(\widehat{E} / \mathbb{Q})[\psi]=0$ implies $\widehat{C}=0$, hence $\amalg(E / \mathbb{Q})[2]=$ $\amalg(E / \mathbb{Q})[\phi]$ in this case.

Proposition 9. If $k=p l$ is a product of primes $p \equiv l \equiv 1 \bmod 8$ with $(p / l)=-1$, then

$$
\amalg(E / \mathbb{Q})[\phi]=\langle 2, p l\rangle \quad \text { whenever } \quad(-4 / p)_{8}(-4 / l)_{8}=-1 .
$$

If this condition holds, then $\# \mathbf{W}(E / \mathbb{Q})[2]=4$.

Proof. Consider $\mathcal{T}^{(\phi)}(2): N^{2}=2 M^{4}+2 p^{2} l^{2} e^{4}$.

- Assume first that $(M, p l)=1$; then $N=2 n$ gives $2 n^{2}=M^{4}+p^{2} l^{2} e^{4}$. Now $M^{2}+p l e^{2} i \equiv 1+i \bmod 8$ and unique factorization in $\mathbb{Z}[i]$ shows that $M^{2}+p l e^{2} i=(1+i) \nu^{2}$. Write $p=\pi \bar{\pi}$ for primes $\pi, \bar{\pi} \equiv 1 \bmod 2+2 i$; reducing modulo $\pi$ gives $[1+i / \pi]=+1$, that is, $(-4 / p)_{8}=+1$, and similarly $(-4 / l)_{8}=+1$.

- If $(M, p l)=p$, put $M=m p$ and $N=2 p n$; then we get $2 n^{2}=$ $\left(p m^{2}+l e^{2} i\right)\left(p m^{2}+l e^{2} i\right)$, and again $p m^{2}+l e^{2} i=(1+i) \nu^{2}$. Reducing modulo $\pi$ gives $[1+i / \pi]=[l / \pi]=(l / p)=-1$, hence $(-4 / p)_{8}=(-4 / l)_{8}=-1$.

- The cases $(M, p l)=l$ and $(M, p l)=p l$ are treated similarly.

Next take $\mathcal{T}^{(\phi)}(p l): N^{2}=p l M^{4}+4 p l e^{4}$. With $N=p l n$ this gives $p l n^{2}=M^{4}+4 e^{4}$; since we may switch the roles of $M$ and $e$ we may assume that $M$ is odd and $e$ is even. Reducing modulo $p$ and $l$ shows that $(-4 / p l)_{8}=$ $(M e / p)$. Write $e=2^{j} e^{\prime}$ with $e^{\prime}$ odd; then $(e / p)=\left(e^{\prime} / p\right)=\left(p / e^{\prime}\right)=1$ and $(M / p)=(p / M)=1$. Thus $(-4 / p l)_{8}=1$.

Finally look at $\mathcal{T}^{(\phi)}(2 p l): 2 p l n^{2}=M^{4}+e^{4}$. As above, $M^{2}+i e^{2}=$ $(1+i) \pi \lambda \nu^{2}$; adding this equation to its conjugate gives $2 M^{2}=(1+i) \pi \lambda \nu^{2}+$ $(1-i) \bar{\pi} \bar{\lambda} \bar{\nu}^{2}$. Reducing modulo $\bar{\pi}$ gives $1=(2 / p)=[1+i / \bar{\pi}][\pi / \bar{\pi}][\lambda / \bar{\pi}]$. Now $[\pi / \bar{\pi}]=1$ and $[\lambda / \bar{\pi}]=[\lambda / \pi]$, hence $(-4 / p)_{8}=[\pi / \lambda]$. Similarly, $(-4 / l)_{8}=$ $[\pi / \lambda]$, and the claim follows. Note that $[\pi / \lambda]$ depends on the choice of $\pi$ and $\lambda$.

From Proposition 9, by a standard application of Chebotarev's density theorem we get

Corollary 10. The curves of rank 0 among $E_{p l}$, where $p \equiv l \equiv 1 \bmod 8$ are primes such that $(p / l)=-1$, have density at least $1 / 2$.

4.2. The case $(p / l)=+1$. Let $k=p l$ be a product of primes $p \equiv l \equiv$ $1 \bmod 8$ with $(p / l)=+1$. Then (see [20])

$$
\operatorname{Sel}^{(\psi)}(\widehat{E} / \mathbb{Q})=\langle-1, p, l\rangle, \quad \operatorname{Sel}^{(\phi)}(E / \mathbb{Q})=\langle 2, p, l\rangle .
$$

Moreover $\langle-1, p l\rangle \subseteq W(\widehat{E} / \mathbb{Q})$. As above, we will now compute non-trivial elements of $\amalg(E / \mathbb{Q})[\phi]$ and $\mathbf{\amalg}(\widehat{E} / \mathbb{Q})[\psi]$. 
The $\psi$-part

First we observe that $W(\widehat{E} / \mathbb{Q})$ always contains $\langle-1, p l\rangle$. Thus either

$$
\begin{aligned}
& W(\widehat{E} / \mathbb{Q})=\langle-1, p, l\rangle \quad \text { and } \quad \amalg(\widehat{E} / \mathbb{Q})[\psi]=0, \quad \text { or } \\
& W(\widehat{E} / \mathbb{Q})=\langle-1, p l\rangle \quad \text { and } \quad \amalg(\widehat{E} / \mathbb{Q})[\psi]=\langle p\rangle \text {, }
\end{aligned}
$$

where $\langle p\rangle$ represents the class of $p \mathbb{Q}^{\times 2}$ (which is the same as the class of $l \mathbb{Q}^{\times 2}$ in view of $\left.p l \mathbb{Q}^{\times 2} \in W(\widehat{E} / \mathbb{Q})\right)$ in $\amalg(\widehat{E} / \mathbb{Q})[\psi]$.

It is therefore sufficient to consider the torsor $\mathcal{T}^{(\psi)}(p): N^{2}=p M^{4}-$ $p l^{2} e^{4}$. Here the right hand side factors over $\mathbb{Q}$ as $N^{2}=p\left(M^{2}-l e^{2}\right)\left(M^{2}+l e^{2}\right)$. We have the following possibilities concerning divisibility:

by $2:\left\{\begin{array}{l}\text { 1) } 2 \mid e, 2 \nmid M N, \\ 2) 2 \mid N, 2 \nmid M e,\end{array} \quad\right.$ by $l:\left\{\begin{array}{l}\text { A) } l \nmid M N, \\ \text { B) } l|M, l| N,\end{array} \quad\right.$ by $p:\left\{\begin{array}{l}\text { a) } p \mid\left(M^{2}+l e^{2}\right), \\ \text { b) } p \mid\left(M^{2}-l e^{2}\right)\end{array}\right.$

Thus we have to consider eight different cases.

Proposition 11. Let $E$ be the elliptic curve defined by $y^{2}=x\left(x^{2}-k^{2}\right)$, where $k=p l$ and where $p \equiv l \equiv 1 \bmod 8$ are primes such that $(p / l)=1$. If the torsor

$$
\mathcal{T}^{(\psi)}(p): \quad N^{2}=p M^{4}-p l^{2} e^{4},
$$

has a rational solution, then the conditions in Table 2 hold according to the case we are in.

Table 2. Let $p \equiv l \equiv 1 \bmod 8$ be primes such that $(p / l)=1$. If $\mathcal{T}^{(\psi)}(p)$ has a rational point, then the conditions $(*)$ hold.

\begin{tabular}{ll}
\hline Case & \multicolumn{1}{c}{ Conditions $(*)$} \\
\hline $1 \mathrm{Aa})$ & {$[\Pi / \Lambda]=(l / p)_{4}=(-4 / p)_{8}=1$} \\
$1 \mathrm{Ab})$ & {$[\Pi / \Lambda]=(p / l)_{4}=(l / p)_{4}(-4 / p)_{8}=1$} \\
$1 \mathrm{Ba})$ & {$[\Pi / \Lambda]=(-4 / p l)_{8},(l / p)_{4}=(p / l)_{4}(-4 / p)_{8}=1$} \\
$1 \mathrm{Bb})$ & {$[\Pi / \Lambda]=(-4 / l)_{8},(p / l)_{4}=(-4 / p)_{8}=1$} \\
$2 \mathrm{Aa})$ & {$[\Pi / \Lambda]=(-4 / p)_{8},(l / p)_{4}=(-4 / l)_{8}=1$} \\
$2 \mathrm{Ab})$ & {$[\Pi / \Lambda]=(l / p)_{4}=(p / l)_{4}(-4 / l)_{8}=1$} \\
$2 \mathrm{Ba})$ & {$[\Pi / \Lambda]=(-4 / p l)_{8},(p / l)_{4}=(l / p)_{4}(-4 / l)_{8}=1$} \\
$2 \mathrm{Bb})$ & {$[\Pi / \Lambda]=(p / l)_{4}=(-4 / l)_{8}=1$} \\
\hline
\end{tabular}

If we are in case 1A), then putting $N=p n$ in (1) gives $p n^{2}=M^{4}-l^{2} e^{4}=$ $\left(M^{2}-l e^{2}\right)\left(M^{2}+l e^{2}\right)$. In case 1Aa), these two factors are coprime, hence $M^{2}+l e^{2}=p a^{2}$ (I) and $M^{2}-l e^{2}=b^{2}$ (II), where $a b=n$. By adding and subtracting (I) and (II) we get $2 M^{2}=b^{2}+p a^{2}$ (III) and $2 l e^{2}=p a^{2}-b^{2}$ (IV). In a similar way we find the following table displaying the four equations I-IV whose solvability follows from the existence of a rational point on (1): 


\begin{tabular}{lcccc}
\hline Case & I & II & III & IV \\
\hline $1 \mathrm{Aa})$ & $M^{2}+l e^{2}=p a^{2}$ & $M^{2}-l e^{2}=b^{2}$ & $2 M^{2}=b^{2}+p a^{2}$ & $2 l e^{2}=p a^{2}-b^{2}$ \\
$1 \mathrm{Ab})$ & $M^{2}+l e^{2}=a^{2}$ & $M^{2}-l e^{2}=p b^{2}$ & $2 M^{2}=a^{2}+p b^{2}$ & $2 l e^{2}=a^{2}-p b^{2}$ \\
$1 \mathrm{Ba})$ & $l m^{2}+e^{2}=p a^{2}$ & $l m^{2}-e^{2}=b^{2}$ & $2 l m^{2}=b^{2}+p a^{2}$ & $2 e^{2}=p a^{2}-b^{2}$ \\
$1 \mathrm{Bb})$ & $l m^{2}+e^{2}=a^{2}$ & $l m^{2}-e^{2}=p b^{2}$ & $2 l m^{2}=a^{2}+p b^{2}$ & $2 e^{2}=a^{2}-p b^{2}$ \\
$2 \mathrm{Aa})$ & $M^{2}+l e^{2}=2 p a^{2}$ & $M^{2}-l e^{2}=2 b^{2}$ & $M^{2}=b^{2}+p a^{2}$ & $l e^{2}=p a^{2}-b^{2}$ \\
$2 \mathrm{Ab})$ & $M^{2}+l e^{2}=2 a^{2}$ & $M^{2}-l e^{2}=2 p b^{2}$ & $M^{2}=a^{2}+p b^{2}$ & $l e^{2}=a^{2}-p b^{2}$ \\
$2 \mathrm{Ba})$ & $l m^{2}+e^{2}=2 p a^{2}$ & $l m^{2}-e^{2}=2 b^{2}$ & $l m^{2}=b^{2}+p a^{2}$ & $e^{2}=p a^{2}-b^{2}$ \\
$2 \mathrm{Bb})$ & $l m^{2}+e^{2}=2 a^{2}$ & $l m^{2}-e^{2}=2 p b^{2}$ & $l m^{2}=a^{2}+p b^{2}$ & $e^{2}=a^{2}-p b^{2}$ \\
\hline
\end{tabular}

In order to save some work we prove a general result that may be applied to each of these cases:

Proposition 12. Let $A, B, C, D \in \mathbb{N}$ be pairwise coprime integers, each a product of distinct primes $\equiv 1 \bmod 4$, and assume that these primes are quadratic residues of each other. If there are $x, y, v, w \in \mathbb{N}$ such that

$$
\begin{aligned}
& A x^{2}+B y^{2}=C v^{2}, \\
& A x^{2}-B y^{2}=D w^{2},
\end{aligned}
$$

then $C \equiv D \bmod 8$, and $A, B, C$ and $D$ satisfy the relations

$$
\left(\frac{A B}{C}\right)_{4}\left(\frac{A D}{B}\right)_{4}\left(\frac{B D}{A}\right)_{4}=1
$$

and

$$
(-1)^{(C-D) / 8}\left(\frac{2}{C D}\right)_{4}\left(\frac{B C}{D}\right)_{4}\left(\frac{B D}{C}\right)_{4}\left(\frac{C D}{A}\right)_{4}=1 .
$$

Proof. Assume that we have a congruence $A r^{2} \equiv B s^{2} \bmod D$ with $(r, D)$ $=(s, D)=1$, and that $(A B / p)=+1$ for all $p \mid D$. Then for each such $p$ we have $A r^{2} \equiv B s^{2} \bmod p$, and raising this congruence to the $(p-1) / 4$ th power we find that $(A / p)_{4}(r / p)=(B / p)_{4}(s / p)$; multiplying these relations together shows that $(A B / D)_{4}=(r s / D)$. We will use this type of reasoning without comment below.

We may (and will) assume that $(x, y)=1$. From $2 y^{2} \equiv 2 B y^{2}=C v^{2}-$ $D w^{2} \equiv v^{2}-w^{2} \bmod 4$ we then deduce that $2 \mid y$ and $2 \nmid x v w$.

Reducing (2) modulo $C$ gives $(-A B / C)_{4}=(x y / C)$. Writing $y=2^{j} y^{\prime}$ for some odd $y^{\prime}$ gives $(y / C)=(2 / C)^{j}\left(y^{\prime} / C\right)=(2 / C)^{j}\left(C / y^{\prime}\right)$. Reducing (2) modulo $y^{\prime}$ we see that $\left(C / y^{\prime}\right)=\left(A / y^{\prime}\right)$. Similarly, we get $(x / C)=(C / x)=$ $(B / x)=(x / B)$ from $(2)$, and $(x / B)=(A D / B)_{4}(w / B)$. Since $(w / B)=$ $(B / w)=(A / w)=(w / A)=(-B D / A)_{4}(y / A)=(-B D / A)_{4}(2 / A)^{j}\left(A / y^{\prime}\right)$, collecting our results gives the relation $(-A B / C)_{4}=(A D / B)_{4}(-B D / A)_{4}$ $\times(2 / A C)^{j}$. Next we have $(-1 / A)_{4}=(2 / A)$ and $(-1 / C)=(2 / C)$, hence the relation becomes $(A B / C)_{4}(A D / B)_{4}(B D / A)_{4}=(2 / A C)^{j+1}$. 
Now there are two cases: if $j=1$, then $A \equiv C+4 \bmod 8$, hence $(2 / A C)=$ -1 , but $(2 / A C)^{j+1}=1$; if $j \geq 2$, then $A \equiv C \bmod 8$, hence $(2 / A C)=1$. In both cases, we arrive at the desired relation.

By adding and subtracting (2) and (3), we get

$$
\begin{aligned}
& 2 A x^{2}=C v^{2}+D w^{2}, \\
& 2 B y^{2}=C v^{2}-D w^{2} .
\end{aligned}
$$

From (7) and the fact that $y$ is even we deduce that $C \equiv D \bmod 8$.

Reducing (7) modulo $D$ yields $(2 B C / D)_{4}=(v y / D)$. From $(7)$ we deduce that $(y / D)=(2 / D)^{j}\left(y^{\prime} / D\right)=(2 / D)^{j}\left(D / y^{\prime}\right)=(2 / D)^{j}\left(C / y^{\prime}\right)$ and $(v / D)=$ $(D / v)=(2 A / v)$, so $(2 B C / D)_{4}=(2 A / v)(2 / D)^{j}\left(C / y^{\prime}\right)$. Similarly, we have $(-2 B D / C)_{4}=(w y / C),(y / C)=(2 / C)^{j}\left(C / y^{\prime}\right)$ and $(w / C)=(C / w)=$ $(2 A / w)$. Combining these results yields the relation $(2 B C / D)_{4}(-2 B D / C)_{4}$ $=(2 A / v w)(2 / C D)^{j}$. Since $C \equiv D \bmod 8$, we have $(2 / C D)=1$, and using $(-1 / C)_{4}=(2 / C)$ we conclude that

$$
\left(\frac{2 B C}{D}\right)_{4}\left(\frac{2 B D}{C}\right)_{4}=\left(\frac{2 A}{v w}\right)\left(\frac{2}{C}\right) .
$$

Next, $(A / w)=(w / A)=(-B D / A)_{4}(y / A)$ and $(A / v)=(v / A)=$ $(B C / A)_{4}(y / A)$, thus $(A / v w)=(-B D / A)_{4}(B C / A)_{4}=(2 / A)(C D / A)_{4}$ since $(B / A)=+1$. This gives us

$$
\left(\frac{2}{C D}\right)_{4}\left(\frac{B C}{D}\right)_{4}\left(\frac{B D}{C}\right)_{4}\left(\frac{C D}{A}\right)_{4}=\left(\frac{2}{v w}\right)\left(\frac{2}{C}\right) .
$$

If $j=1$, then $C v^{2} \equiv D w^{2}+8 \bmod 16$, hence $C \equiv D+8 \bmod 16$ if and only if $(2 / v)=(2 / w)$, or $(2 / v w)=-(-1)^{(C-D) / 8}$. Moreover, $2 A x^{2} \equiv 2 C v^{2}+$ 8 mod 16 implies $(2 / A C)=-1$, so we get $(2 / v w)(2 / A C)=(-1)^{(C-D) / 8}$.

If $j \geq 2$, then $C v^{2} \equiv D w^{2} \bmod 16$, and this shows that $C \equiv D \bmod 16$ if and only if $(2 / v)=(2 / w)$, hence $(2 / v w)=(-1)^{(C-D) / 8}$. Moreover, (6) implies that $A \equiv C \bmod 8$, hence $(2 / A C)=+1$, and again $(2 / v w)(2 / A C)=$ $(-1)^{(C-D) / 8}$.

In order to apply this result we have to identify the coefficients $A, B, C$ and $D$. We find

\begin{tabular}{lcccccc|ccccccc}
\hline Case & $(1)$ & $(2)$ & $A$ & $B$ & $C$ & $D$ & Case & $(1)$ & $(2)$ & $A$ & $B$ & $C$ & $D$ \\
\hline $1 \mathrm{Aa})$ & I & II & 1 & $l$ & $p$ & 1 & $2 \mathrm{Aa})$ & III & IV & $p$ & 1 & 1 & $l$ \\
$1 \mathrm{Ab})$ & I & II & 1 & $l$ & 1 & $p$ & $2 \mathrm{Ab})$ & III & IV & 1 & $p$ & 1 & $l$ \\
$1 \mathrm{Ba})$ & I & II & $l$ & 1 & $p$ & 1 & $2 \mathrm{Ba})$ & III & IV & $p$ & 1 & $l$ & 1 \\
$1 \mathrm{Bb})$ & I & II & $l$ & 1 & 1 & $p$ & $2 \mathrm{Bb})$ & III & IV & 1 & $p$ & $l$ & 1 \\
\hline
\end{tabular}

This takes care of all the conditions not involving $[\Pi / \Lambda]$. To complete the proof we need the following 
Lemma 13. Let $P \equiv L \equiv 1 \bmod 8$ be primes such that $(P / L)=+1$. Let $\Pi, \Lambda \in \mathbb{Z}[\sqrt{2}]$ be primary elements of norm $P$ and $L$, respectively. If there exist integers $x, y, z, w \in \mathbb{N}$ such that

$$
x^{2}-2 y^{2}=-P z^{2} \quad \text { and } \quad x^{2}-y^{2}=\epsilon L w^{2}
$$

for some $\epsilon= \pm 1$, then $[\Pi / \Lambda]=+1$.

Proof. Unique factorization gives $x+y \sqrt{2}=\varepsilon_{2} \Pi \alpha^{2}$, where $\varepsilon_{2}$ is a fundamental unit of $\mathbb{Z}[\sqrt{2}]$ and where $N \alpha=z$. Thus $[\Pi / \Lambda]=\left[\varepsilon_{2} / \Lambda\right][x+y \sqrt{2} \Lambda]$. Now $y \equiv \pm x \bmod \Lambda$ from the second equation, which yields $[x+y \sqrt{2} / \Lambda]=$ $[x / \Lambda][1 \pm \sqrt{2} / \Lambda]$. But $[1 \pm \sqrt{2} / \Lambda]=\left[\varepsilon_{2} / \Lambda\right]$ since the expression $[ \pm 1 \pm \sqrt{2} / \Lambda]$ does not depend on the choice of signs, and we get $[\Pi / \Lambda]=[x / \Lambda]=(x / L)$. If $\epsilon=+1$, then $(x / L)=(y / L)=(L / y)=+1$, and if $\epsilon=-1$, then $(x / L)=(L / x)=+1$. This proves our claim.

Lemma 13 takes care of four out of our eight cases:

\begin{tabular}{cccccccc}
\hline Case & $x$ & $y$ & $z$ & $w$ & $P$ & $L$ & $\epsilon$ \\
\hline $1 \mathrm{Aa})$ & $b$ & $M$ & $a$ & $e$ & $p$ & $l$ & -1 \\
$1 \mathrm{Ab})$ & $a$ & $M$ & $b$ & $e$ & $p$ & $l$ & +1 \\
$2 \mathrm{Ab})$ & $M$ & $a$ & $e$ & $b$ & $l$ & $p$ & +1 \\
$2 \mathrm{Bb})$ & $e$ & $a$ & $m$ & $b$ & $l$ & $p$ & -1 \\
\hline
\end{tabular}

For the remaining four cases, the role of Lemma 13 is taken over by

Lemma 14. Let $P \equiv L \equiv 1 \bmod 8$ be primes such that $(P / L)=+1$. Let $\Pi, \Lambda \in \mathbb{Z}[\sqrt{2}]$ be primary elements of norm $P$ and $L$, respectively. If there exist integers $x, y, z, w \in \mathbb{N}$ such that

$$
x^{2}+2 \epsilon y^{2}=P z^{2} \quad \text { and } \quad x^{2}+\epsilon y^{2}=L w^{2}
$$

for some $\epsilon= \pm 1$, then

$$
\left[\frac{\Pi}{\Lambda}\right]= \begin{cases}(-4 / L)_{8} & \text { if } \epsilon=-1 \\ (P / L)_{4}(L / P)_{4}(-4 / L)_{8} & \text { if } \epsilon=+1 .\end{cases}
$$

Proof. Let $\pi, \lambda \in \mathbb{Z}[\sqrt{2 \epsilon}]$ be primary elements of norm $P$ and $L$, respectively. Then from $\pi \alpha^{2}=x+y \sqrt{2 \epsilon}$ we get $[\pi / \lambda]=[x+y \sqrt{2 \epsilon} / \lambda]$. The second equation gives $x \equiv \pm y \sqrt{\epsilon} \bmod \mathfrak{l}$, where $\mathfrak{l}$ denotes a prime ideal above $l$ in $\mathbb{Q}\left(\zeta_{8}\right)$. Letting $\{\cdot / \cdot\}$ denote the quadratic residue symbol in $\mathbb{Z}\left[\zeta_{8}\right]$, we find

$$
[x+y \sqrt{2 \epsilon} / \lambda]=\{x+y \sqrt{2 \epsilon} / \mathfrak{l}\}=\{x \pm x \sqrt{2} / \mathfrak{l}\}=(x / L)[1 \pm \sqrt{2} / \Lambda] .
$$

Now if $\epsilon=1$ then $(x / L)=(L / x)=+1$, whereas if $\epsilon=-1$ then $(x / L)=$ $(y / L)=\left(y^{\prime} / L\right)=\left(L / y^{\prime}\right)=+1$. Thus $[\pi / \lambda]=[1+\sqrt{2} / \Lambda]=(-4 / L)_{8}$. If $\epsilon=-1$, then $\pi=\Pi$ and $\lambda=\Lambda$, but if $\epsilon=+1$ then $\pi=\Pi^{*}$ and $\lambda=\Lambda^{*}$, $\Pi^{*}, \Lambda^{*} \in \mathbb{Z}[\sqrt{-2}]$ are primary elements of norm $p$ and $l$, respectively. Thus $[\Pi / \Lambda]=\left[\Pi^{*} / \Lambda^{*}\right](P / L)_{4}(L / P)_{4}=(P / L)_{4}(L / P)_{4}(-4 / L)_{8}$. 
Lemma 14 covers the remaining four cases:

\begin{tabular}{ccccccccl}
\hline Case & $x$ & $y$ & $z$ & $w$ & $P$ & $L$ & $\epsilon$ & Resulting condition \\
\hline $1 \mathrm{Ba})$ & $b$ & $e$ & $a$ & $m$ & $p$ & $l$ & +1 & {$[\Pi / \Lambda]=(-4 / p l)_{8}$} \\
$1 \mathrm{Bb})$ & $a$ & $e$ & $m$ & $b$ & $p$ & $l$ & -1 & {$[\Pi / \Lambda]=(-4 / l)_{8}$} \\
$2 \mathrm{Aa})$ & $M$ & $b$ & $a$ & $e$ & $l$ & $p$ & -1 & {$[\Pi / \Lambda]=(-4 / p)_{8}$} \\
$2 \mathrm{Ba})$ & $e$ & $b$ & $m$ & $a$ & $l$ & $p$ & +1 & {$[\Pi / \Lambda]=(-4 / p l)_{8}$} \\
\hline
\end{tabular}

Note that in case $1 \mathrm{Ba})$, Lemma 14 gives $[\Pi / \Lambda]=(-4 / l)_{8}(p / l)_{4}(l / p)_{4}$; but since $(p / l)_{4}(l / p)_{4}=(-4 / p)_{8}$ by Lemma 13 , we get the relation in the table above.

As a matter of fact, the criteria involving $[\Pi / \Lambda]$ can just as well be obtained using genus theory (compare the discussion of $\mathcal{T}^{(\phi)}(2 p)$ below). As the discussion of the $\phi$-part below shows, however, it seems that arguments from genus theory cannot always be replaced by the direct calculation of residue symbols.

$$
\text { The } \phi \text {-part }
$$

Our aim in this section is to show

Proposition 15. If the torsor $\mathcal{T}^{(\phi)}\left(b_{1}\right)$ with $1 \neq b_{1} \in\langle 2, p, l\rangle$ has a rational point, then the conditions in Table 3 must be satisfied.

Table 3. Let $p \equiv l \equiv 1 \bmod 8$ be primes such that $(p / l)=1$. If $\mathcal{T}^{(\phi)}\left(b_{1}\right)$ has a rational point, then the conditions $(*)$ must be satisfied.

\begin{tabular}{ll}
\hline$b_{1}$ & \multicolumn{1}{c}{ Conditions $(*)$} \\
\hline 2 & $(-4 / p)_{8}=(-4 / l)_{8}=[\Pi / \Lambda]=1$ \\
$p$ & $(p / l)_{4}=(l / p)_{4}=(-4 / p)_{8}=1$ \\
$2 p$ & $(p / l)_{4}(l / p)_{4}=(-4 / l)_{8},(-4 / p)_{8}=1,[\Pi / \Lambda]=(l / p)_{4}$ \\
$l$ & $(p / l)_{4}=(l / p)_{4}=(-4 / l)_{8}=1$ \\
$2 l$ & $(p / l)_{4}(l / p)_{4}=(-4 / p)_{8},(-4 / l)_{8}=1,[\Pi / \Lambda]=(p / l)_{4}$ \\
$p l$ & $(p / l)_{4}=(l / p)_{4},(-4 / p)_{8}=(-4 / l)_{8}=1$ \\
$2 p l$ & $(p / l)_{4}(l / p)_{4}=(-4 / p)_{8}=(-4 / l)_{8},[\Pi / \Lambda]=1$ \\
\hline
\end{tabular}

For the proof of Proposition 15, we need the following proposition dealing with a slightly more general situation:

Proposition 16. Let $k$ be a product of pairwise distinct primes $\equiv 1$ mod 8 that are quadratic residues of each other. Let $k=A B$ for $A, B \in \mathbb{N}$; if the torsor $\mathcal{T}^{(\phi)}(A)$ of $E_{k}$ has a non-trivial rational point, then there is a primary $\alpha \in \mathbb{Z}[i]$ with norm $A$ such that the following conditions hold:

(i) $(-4 / A)_{8}=+1$;

(ii) $[\alpha / \pi]=+1$ for all $\pi \mid B$;

(iii) $(-4 / p)_{8}=(B / p)_{4}$ for all $p \mid A$;

(iv) $\left[\alpha^{*} / \pi\right]=+1$ for all $\pi \mid \alpha$, where $\alpha=\alpha^{*} \pi$. 
Proof. We have $\mathcal{T}^{(\phi)}(A): A N^{2}=M^{4}+4 B^{2} e^{4}$; let $b=\operatorname{gcd}(M, B)$ be normalized by $b>0$. Putting $N=b n$ and $M=b m$, we get $A n^{2}=b^{2} m^{4}+$ $4 c^{2} e^{4}$, where $b c=B$. We may assume that $m$ is odd, otherwise we switch the roles of $m$ and $e$. Note that $A \equiv 1 \bmod 8$ implies that $4 \mid e$.

Factoring the right hand side on $\mathbb{Z}[i]$ gives $\alpha \nu^{2}=b m^{2}+2 c i e^{2}$ for some primary $\alpha \in \mathbb{Z}[i]$ with norm $N \alpha=A$. First observe that $\alpha \nu^{2} \equiv b m^{2} \equiv$ $1 \bmod 8$; thus $\alpha$ is congruent to a square modulo 8 , and this implies (i). Moreover, $[\alpha / \pi]=[b / \pi]=(b / p)=+1$ for all $\pi \mid c$ with $N \pi=p$, and similarly $[\alpha / \pi]=1$ for $\pi \mid b$, hence (ii).

Reducing the equation modulo some $\pi \mid \alpha$ gives $[1+i / \pi](c / p)_{4}=(-b / p)_{4}$, hence $(-4 / p)_{8}=(B / p)_{4}$ for all $p \mid A$, and this is (iii).

Finally, subtracting $\alpha \nu^{2}=b m^{2}+2 c i e^{2}$ from its conjugate yields $\alpha \nu^{2}-$ $\bar{\alpha} \bar{\nu}^{2}=4 c i e^{2}$; reducing modulo some $\bar{\pi} \mid \bar{\alpha}$ we get $[\alpha / \bar{\pi}]=(2 c / p)=+1$. Since $[\pi / \bar{\pi}]=+1$, this is equivalent to $\left[\alpha^{*} / \pi\right]=+1$, proving (iv).

Proof of Proposition 15. In the case $\mathcal{T}^{(\phi)}(p)$ we have $A=p$ and $B=l$, so $(-4 / p)_{8}=1$ from Proposition 16(i), $(p / l)_{4}(l / p)_{4}=[\pi / \lambda]=1$ from Proposition 16(ii), $(-4 / p)_{8}=(l / p)_{4}$ from Proposition 16(iii) and no condition from Proposition 16(iv). In this way we find all criteria given in Table 3 except those involving $[\Pi / \Lambda]$. These have to be derived in an ad hoc manner:

- $\mathcal{T}^{(\phi)}(2): 2 n^{2}=M^{4}+p^{2} l^{2} e^{4}$. Write the torsor in the form $-p^{2} l^{2} e^{4}=$ $\left(M^{2}+n \sqrt{2}\right)\left(M^{2}-n \sqrt{2}\right)$. We assume that $(M, p l)=1$; the other cases are treated similarly. Then $M^{2}+n \sqrt{2}=\eta \Pi^{2} \Lambda^{2} \alpha^{4}$ for primes $\Pi, \Lambda \in \mathbb{Z}[\sqrt{2}]$ such that $N \Pi=p, N \Lambda=l$ and $\Pi \equiv \Lambda \equiv 1 \bmod 2$. Moreover, $\eta=\varepsilon^{ \pm 1}$ with $\varepsilon=1+\sqrt{2}$. Adding the last equation to its conjugate gives $2 M^{2}=$ $(\sqrt{2} M)^{2}=\eta \Pi^{2} \Lambda^{2} \alpha^{4}+\bar{\eta} \bar{\Pi}^{2} \bar{\Lambda}^{2} \bar{\alpha}^{4}$. Replacing $M$ by $M \varepsilon$ if necessary we may assume without loss of generality that $\eta=\varepsilon$. Thus $\varepsilon^{-1}(\sqrt{2} M)^{2}=\Pi^{2} \Lambda^{2} \alpha^{4}-\bar{\varepsilon}^{2} \bar{\Pi}^{2} \bar{\Lambda}^{2} \bar{\alpha}^{4}=\left(\Pi \Lambda \alpha^{2}+\bar{\varepsilon} \bar{\Pi} \bar{\Lambda}^{2}\right)\left(\Pi \Lambda \alpha^{2}-\bar{\varepsilon} \bar{\Pi} \bar{\Lambda} \bar{\alpha}^{2}\right)$. Now $\Pi \Lambda \alpha^{2}+\bar{\varepsilon} \bar{\Pi} \bar{\Lambda} \bar{\alpha}^{2} \equiv \sqrt{2} \bmod 2$, hence $\Pi \Lambda \alpha^{2}+\bar{\varepsilon} \bar{\Pi} \bar{\Lambda} \bar{\alpha}^{2}=\sqrt{2} \mu^{2}, \Pi \Lambda \alpha^{2}-$ $\bar{\varepsilon} \bar{\Pi} \bar{\Lambda}^{2}=\sqrt{2} \varepsilon^{-1} \bar{\mu}^{2}$. Reducing modulo $\bar{\Pi}$ and using $[\Pi / \bar{\Pi}]=(2 / p)_{4},[\varepsilon / \bar{\Pi}]$ $=(-4 / p)_{8}=1$ (in this case), as well as $[\Lambda / \bar{\Pi}]=[\Lambda / \Pi]$ we find that the solvability of $\mathcal{T}^{(\phi)}(2)$ implies $[\Lambda / \Pi]=1$.

- $\mathcal{T}^{(\phi)}(2 p)$ : Factoring $2 p n^{2}=M^{4}+l^{2} e^{4}$ as $2 p n^{2}=\left(M^{2}+l e^{2}+M e \sqrt{2 l}\right)$ $\times\left(M^{2}+l e^{2}-M e \sqrt{2 l}\right)$ and observing that $M e \equiv 1 \bmod 2$ implies that each factor is divisible exactly once by the prime ideal 2 above 2 . Thus $2 \mathfrak{p n}^{2}=$ $\left(M^{2}+l e^{2}+M e \sqrt{2 l}\right)$, where $\mathfrak{n}$ is an ideal with norm $n$. Let $h^{+}$denote the class number of $\mathbb{Q}(\sqrt{2 l})$ in the strict sense. We have to distinguish several cases:

(1) $h \equiv 2 \bmod 4, h^{+} \equiv 4 \bmod 8$. By Proposition 3, this holds if and only if $(-4 / l)_{8}=-1$, and we also know that $N \varepsilon_{2 l}=+1$ and that 2 is principal in the wide sense. Now $[\Pi / \Lambda]=+1 \Leftrightarrow \mathfrak{p} \stackrel{+}{\sim} 4$ by Lemma 4 , and since $2 \mathbf{p n}^{2}$ is principal in the strict sense, this happens if and only if 
$2 \mathfrak{n}^{2} \stackrel{+}{\sim}$. If $(2 / l)_{4}=-1$, then $2 \stackrel{+}{\sim} 1$ is principal in the strict sense, and this happens if and only if $\mathfrak{n}^{2} \stackrel{ \pm}{\sim}$, thus by genus theory $\Leftrightarrow(2 / n)=(l / n)=$ +1 . But $(2 / n)=(2 p / l)_{4}=-(p / l)_{4}$. Finally, solvability of $\mathcal{T}^{(\phi)}(2 p)$ implies $(-4 / l)_{8}=(p / l)_{4}(l / p)_{4}$, so $(p / l)_{4}=(-4 / l)_{8}(l / p)_{4}=-(l / p)_{4}$, and we see that $[\Pi / \Lambda]=(l / p)_{4}$ as claimed. If $(2 / l)_{4}=+1$, on the other hand, then 2 is not principal in the strict sense, hence $[\Pi / \Lambda]=+1 \Leftrightarrow \mathfrak{n}^{2} \nsim 4$, that is, iff $-1=(2 / n)=(p / l)_{4}$, and as above this gives $[\Pi / \Lambda]=(l / p)_{4}$.

(2) $h \equiv h^{+} \equiv 4 \bmod 8$. By Proposition 3 , this holds if and only if $(2 / l)_{4}=-1$ and $l \equiv 9 \bmod 16$. Here $2^{2}$ is principal in the strict sense and 2 is not, in particular $2 \stackrel{+}{\sim} 2$ but $2 \stackrel{+}{\nsim} 4$. Now $[\Pi / \Lambda]=+1 \Leftrightarrow \mathfrak{n} \stackrel{+}{\nsim} 2$ which in turn happens iff $-1=(2 / n)=(2 p / l)_{4}=-(p / l)_{4}$. Since $1=$ $(-4 / l)_{8}=(p / l)_{4}(l / p)_{4}$ from earlier solvability results, this gives $[\Pi / \Lambda]=1$ $\Leftrightarrow(l / p)_{4}=1$ as claimed.

(3) $h^{+} \equiv 0 \bmod 8$. By Proposition 3, this holds if and only if $(2 / l)_{4}=+1$ and $l \equiv 1 \bmod 16$. Here $2^{2}=(2)$ is principal, and since the class group $\mathrm{Cl}_{2}^{+}(k)$ is cyclic, $2 \stackrel{+}{\sim} 4$. Thus $[\Pi / \Lambda]=+1 \Leftrightarrow \mathfrak{n} \stackrel{+}{\sim} 2 \Leftrightarrow 1=(2 p / l)_{4}=$ $(p / l)_{4}$, and we conclude as above that $[\Pi / \Lambda]=(l / p)_{4}$.

- $\mathcal{T}^{(\phi)}(2 l): N^{2}=2 l M^{4}+2 p^{2} l e^{4}$. Symmetry reduces this to the discussion of $\mathcal{T}^{(\phi)}(2 p)$.

- $\mathcal{T}^{(\phi)}(2 p l): N^{2}=2 p l M^{4}+2 p l e^{4}$. We start by factoring the torsor as $2 p l n^{2}=M^{4}+e^{4}=\left(M^{2}+e^{2}+M e \sqrt{2}\right)\left(M^{2}+e^{2}-M e \sqrt{2}\right)$. Unique factorization in $\mathbb{Z}[\sqrt{2}]$ gives $M^{2}+e^{2}+M e \sqrt{2}=\varepsilon \sqrt{2} \Pi \Lambda \nu^{2}$ and $M^{2}+e^{2}-M e \sqrt{2}=$ $-\bar{\varepsilon} \sqrt{2} \bar{\Pi} \bar{\Lambda}^{2}$. Subtracting the second equation from the first gives $2 M e=$ $\varepsilon \Pi \Lambda \nu^{2}+\bar{\varepsilon} \bar{\Pi} \bar{\Lambda} \bar{\nu}^{2}$, which in view of $[\varepsilon / \bar{\Pi}]=(-4 / p)_{8}$ and $[\Pi / \bar{\Pi}]=(2 / p)_{4}$ gives $[\Lambda / \Pi]=(M e / p)(-1 / p)_{8}$.

On the other hand we have $2 p l n^{2}=\left(M^{2}+i e^{2}\right)\left(M^{2}-i e^{2}\right)$, hence $M^{2}+$ $i e^{2}=(1+i) \pi \lambda \nu^{2}$ for some $\nu \in \mathbb{Z}[i]$. This implies $(M e / p)=[M e / \pi]=$ $[-i / \pi]_{4}=(-1 / p)_{8}$, hence our claim that $[\Pi / \Lambda]=1$ is proved.

The use of genus theory in this connection was suggested by the proofs of Pépin's conjectures in [21]. This concludes our discussion of the $\phi$-part of $\amalg(E / \mathbb{Q})$.

5. The main result. The main result of this paper is the following theorem:

Theorem 17. Let $p \equiv l \equiv 1 \bmod 8$ be primes with $(p / l)=1$. The properties of the Tate-Shafarevich groups $\mathbf{\amalg}\left(E_{k} / \mathbb{Q}\right)[\phi]$ and $\mathbf{\amalg}\left(\widehat{E}_{k} / \mathbb{Q}\right)[\psi]$ corresponding to the 2-isogenies between the elliptic curves $E_{k}: y^{2}=x\left(x^{2}-p^{2} l^{2}\right)$ and $\widehat{E}_{k}: y^{2}=x\left(x^{2}+4 p^{2} l^{2}\right)$ are recorded in Table 4 . If the rank given there is 0 , then the given subgroups actually equal $\amalg\left(E_{k} / \mathbb{Q}\right)[\phi]$ and $\amalg\left(\widehat{E}_{k} / \mathbb{Q}\right)[\psi]$, and we have $\amalg(E / \mathbb{Q})[2] \simeq(\mathbb{Z} / 2 \mathbb{Z})^{4}$. 
Table 4. The Tate-Shafarevich groups $\amalg[\phi]:=\amalg\left(E_{k} / \mathbb{Q}\right)[\phi]$ and $\amalg[\psi]:=\amalg\left(\widehat{E}_{k} / \mathbb{Q}\right)[\psi]$ corresponding to the 2-isogenies between the elliptic curves $E_{k}: y^{2}=x\left(x^{2}-k^{2}\right)$ and $\widehat{E}_{k}: y^{2}=x\left(x^{2}+4 k^{2}\right)$ with $k=p l$, where $p$ and $l$ are primes such that $p \equiv l \equiv 1 \bmod 8$ and $(p / l)=+1$, have subgroups as indicated. The column labeled rk $E$ gives bounds for the rank of $E_{k}(\mathbb{Q})$. The column $W^{(\phi)}$ gives the subgroup of torsors in $\operatorname{Sel}^{(\phi)}(E / \mathbb{Q})$ that may have rational points.

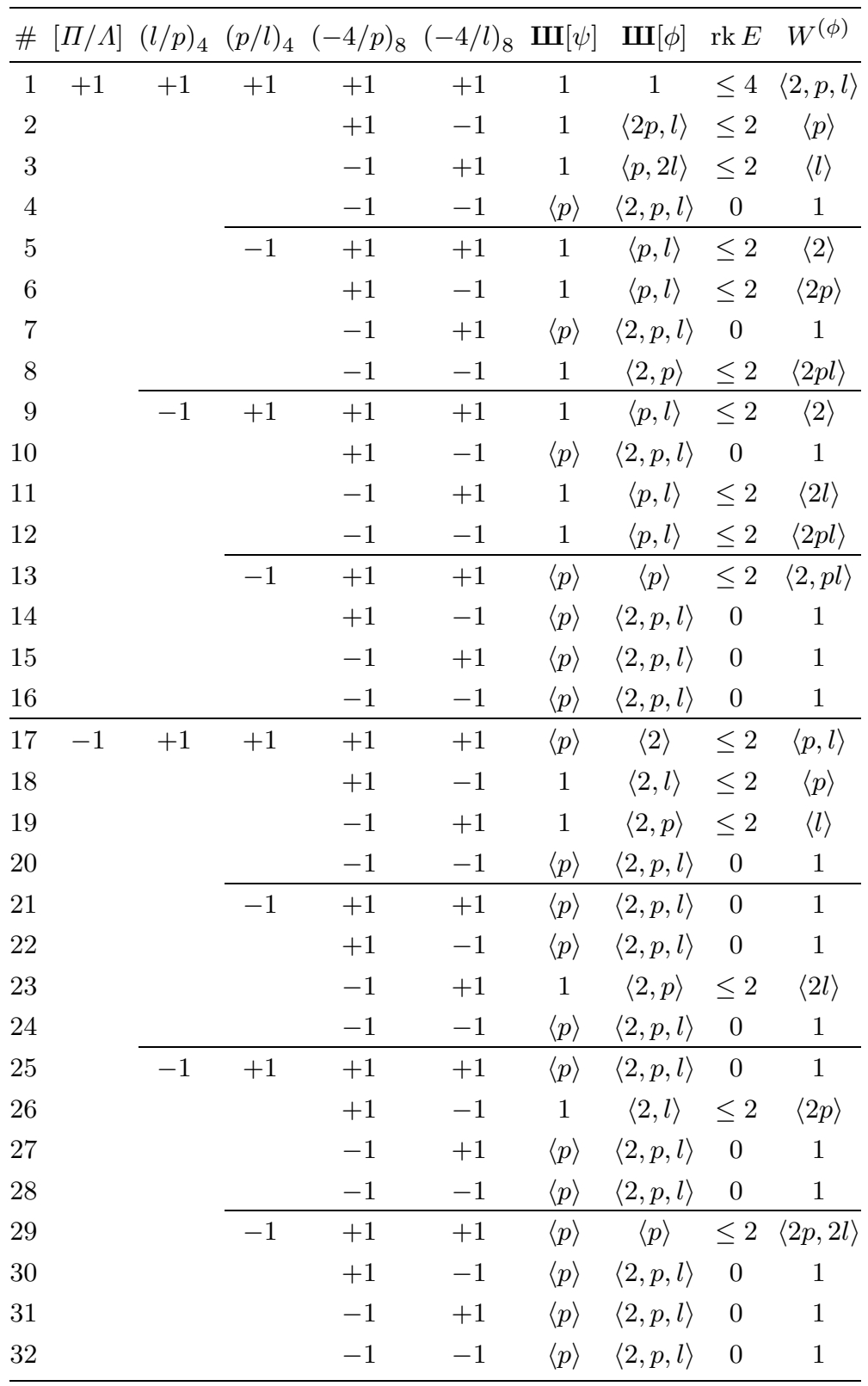


Let us sketch the proof of Theorem 17 by going through an example. Take the second line; we claim that $\mathcal{T}^{(\phi)}(p)$ is the only possibly trivial torsor in $\operatorname{Sel}^{(\phi)}(E / \mathbb{Q})$ (that means that it is the only one that might have a rational point). In fact, the torsors $\mathcal{T}^{(\phi)}(2), \mathcal{T}^{(\phi)}(l), \mathcal{T}^{(\phi)}(2 l)$ and $\mathcal{T}^{(\phi)}(p l)$ are non-trivial since $(-4 / l)_{8}=-1$, whereas $\mathcal{T}^{(\phi)}(2 p)$ and $\mathcal{T}^{(\phi)}(2 p l)$ are non-trivial because $(p / l)_{4}(l / p)_{4} \neq(-4 / l)_{8}$. The other claims now follow immediately.

It remains to prove that $\amalg(E / \mathbb{Q})[2]$ has order 16 if $\operatorname{rank} E_{p l}=0$. Recall the exact sequence

$$
0 \rightarrow \amalg(E / \mathbb{Q})[\phi] \rightarrow \amalg(E / \mathbb{Q})[2] \rightarrow \amalg(\widehat{E} / \mathbb{Q})[\psi] \rightarrow \widehat{C} \rightarrow 0,
$$

where $\widehat{C}$ is a finite 2 -group of even rank by a result of Cassels. Since $\widehat{C}$ is a quotient of the group $\amalg(\widehat{E} / \mathbb{Q})[\psi]$ of order 2 in our case, we must have $\widehat{C}=0$, and in particular $\amalg(E / \mathbb{Q})[2] \simeq \amalg(E / \mathbb{Q})[\phi] \oplus \amalg(\widehat{E} / \mathbb{Q})[\psi]$ as claimed.

CoROllary 18. The curves of rank 0 among $E_{p l}$, where $p \equiv l \equiv 1 \bmod 8$ are primes such that $(p / l)=+1$, have density at least $1 / 2$. Those with rank 4 have density at most $1 / 32$.

Table 5 gives the smallest examples of $p$ and $l$ satisfying the conditions from Table 4 and such that the given inequality for the rank is an equality (with the possible exception of the first line with $p=41, l=2273$, where the rank is 2 or 4 ). In all cases except one, the given example is the one that occurs first; the exception is $p l=41 \cdot 1601$, where the example $p l=41 \cdot 1321$ has the same residue symbols; yet rank $E_{41 \cdot 1321}=0$.

If $E=E_{p l}$ is a curve with $\# \amalg(\widehat{E} / \mathbb{Q})[\psi]=2$, then Proposition 1 and the fact that $\widehat{C}$ has even rank imply that we must have $\widehat{C}=0$; this in turn implies that every element of $\operatorname{Sel}^{(\psi)}(\widehat{E} / \mathbb{Q})$ can be lifted to an element in $\operatorname{Sel}^{(2)}(\widehat{E} / \mathbb{Q})$, in other words: the second 2-descent via 2-isogenies never detects groups $\amalg(\widehat{E} / \mathbb{Q})[\psi]$ of order 2 ; in particular, it never predicts rank 0 in the 16 cases where Table 4 does.

Table 5 compares the rank estimates from Theorem 17 with those produced by Cremona's program mwrank; for the column labeled mwrank $E$ I used $E_{k}: y^{2}=x\left(x^{2}-k^{2}\right)$ as the input, whereas for the other one I used the 2-isogenous curve $E_{-2 k l}: y^{2}=x\left(x^{2}+4 k^{2}\right)$. Although both curves have the same rank, the output differs considerably. The reason is that with $E_{k}$ as the input, mwrank chooses the isogeny with kernel $(k, 0)$ (instead of $(0,0)$ as we did), and the second 2-descent for this pair of curves is (in our examples at least) less powerful than for the pair we have picked. On the other hand, choosing $E_{k}$ for $k=113 \cdot 257$, mwrank produces the correct rank 2, whereas $E_{-2 k}$ does not. While this phenomenon has been observed before (e.g. by Nils Bruin [4]), it seems that this problem should be investigated more closely. 
Table 5. A comparison of Table 4 and mwrank. The column labeled mwrank $E$ gives the bounds for the rank of $E=E_{p l}$ produced by Cremona's program, and similarly mwrank $\widehat{E}$ gives the rank estimate produced when running mwrank on $\widehat{E}=E_{-2 p l}$; combining the lower bounds from these columns with the results from Table 4 gives the entries in column $r$.

\begin{tabular}{rccccc}
\hline$\#$ & mwrank $E$ & mwrank $\widehat{E}$ & $r$ & $p$ & $l$ \\
\hline 1 & $2 \leq r \leq 4$ & $2 \leq r \leq 4$ & $2 \leq r \leq 4$ & 41 & 2273 \\
2 & $2 \leq r \leq 4$ & 2 & 2 & 41 & 769 \\
3 & $2 \leq r \leq 4$ & 2 & 2 & 97 & 353 \\
4 & $0 \leq r \leq 4$ & $0 \leq r \leq 2$ & 0 & 17 & 1361 \\
5 & $2 \leq r \leq 4$ & 2 & 2 & 41 & 113 \\
6 & $2 \leq r \leq 4$ & 2 & 2 & 113 & 233 \\
7 & $0 \leq r \leq 4$ & $0 \leq r \leq 2$ & 0 & 17 & 953 \\
8 & $2 \leq r \leq 4$ & 2 & 2 & 17 & 89 \\
9 & $2 \leq r \leq 4$ & 2 & 2 & 41 & 569 \\
10 & $0 \leq r \leq 4$ & $0 \leq r \leq 2$ & 0 & 41 & 73 \\
11 & $2 \leq r \leq 4$ & 2 & 2 & 17 & 457 \\
12 & $2 \leq r \leq 4$ & 2 & 2 & 17 & 433 \\
13 & $2 \leq r \leq 4$ & $2 \leq r \leq 4$ & 2 & 41 & 1601 \\
14 & $0 \leq r \leq 4$ & $0 \leq r \leq 2$ & 0 & 41 & 449 \\
15 & $0 \leq r \leq 4$ & $0 \leq r \leq 2$ & 0 & 17 & 569 \\
16 & $0 \leq r \leq 4$ & $0 \leq r \leq 2$ & 0 & 17 & 977 \\
17 & 2 & $2 \leq r \leq 4$ & 2 & 113 & 569 \\
18 & 2 & 2 & 2 & 41 & 433 \\
19 & 2 & 2 & 2 & 17 & 353 \\
20 & $0 \leq r \leq 2$ & $0 \leq r \leq 2$ & 0 & 73 & 89 \\
21 & $0 \leq r \leq 2$ & $0 \leq r \leq 2$ & 0 & 41 & 353 \\
22 & $0 \leq r \leq 2$ & $0 \leq r \leq 2$ & 0 & 113 & 241 \\
23 & 2 & 2 & 2 & 17 & 137 \\
24 & $0 \leq r \leq 2$ & $0 \leq r \leq 2$ & 0 & 89 & 97 \\
25 & $0 \leq r \leq 2$ & $0 \leq r \leq 2$ & 0 & 41 & 337 \\
26 & 2 & 2 & 2 & 113 & 401 \\
27 & $0 \leq r \leq 2$ & $0 \leq r \leq 2$ & 0 & 17 & 257 \\
28 & $0 \leq r \leq 2$ & $0 \leq r \leq 2$ & 0 & 73 & 97 \\
29 & 2 & $2 \leq r \leq 4$ & 2 & 113 & 257 \\
30 & $0 \leq r \leq 2$ & $0 \leq r \leq 2$ & 0 & 41 & 241 \\
31 & $0 \leq r \leq 2$ & $0 \leq r \leq 2$ & 0 & 89 & 257 \\
32 & $0 \leq r \leq 2$ & $0 \leq r \leq 2$ & 0 & 17 & 281 \\
\hline & & & & &
\end{tabular}


Some examples. In [40], Wada and Taira (extending previous calculations of Noda \& Wada [30]; see also Nemenzo [27]) computed the rank of most curves $E_{k}$ for $k<40000$. For 20 of these curves, they could only prove that the rank was between 2 and 4 . Exactly 8 out of these 20 numbers have the form $k=p l$ with primes $p \equiv l \equiv 1 \bmod 8$, and for these numbers our results show that the rank is in fact 2 in these cases:

\begin{tabular}{cccccccc}
\hline$k$ & $p$ & \multicolumn{1}{c}{$l$} & $(l / p)_{4}$ & $(p / l)_{4}$ & $(-4 / p)_{8}$ & $(-4 / l)_{8}$ & {$[\Pi / \Lambda]$} \\
\hline 1513 & 17 & 89 & +1 & -1 & -1 & -1 & +1 \\
2329 & 17 & 137 & +1 & -1 & -1 & +1 & -1 \\
4633 & 41 & 113 & +1 & -1 & +1 & +1 & +1 \\
6001 & 17 & 353 & +1 & +1 & -1 & +1 & -1 \\
6953 & 17 & 409 & +1 & +1 & -1 & +1 & -1 \\
7361 & 17 & 433 & -1 & +1 & -1 & -1 & +1 \\
7769 & 17 & 457 & -1 & +1 & -1 & +1 & +1 \\
9809 & 17 & 577 & +1 & -1 & -1 & +1 & -1 \\
\hline
\end{tabular}

We remark in passing that the inequality $\operatorname{rank} E \leq 2$ in these cases follows already from the criteria not involving $[\Pi / \Lambda]$. Moreover, the special case $k=1513$ was discussed by Wada [39].

The tables of Nemenzo [28, 29] contain 70 more values $k=p l<100000$ such that $E_{k}$ has analytic rank 2 and Selmer rank 4 . For 66 of them, the criteria involving the rational residue symbols suffice to show that the rank is at most 2 ; the 4 exceptions are $k=64297=113 \cdot 569,67009=113 \cdot 593$, $93193=41 \cdot 2273$ and $94177=41 \cdot 2297$. For these values of $k$ we find $[\Lambda / \Pi]=-1$ except when $k=93193$.

It would be interesting to compare the results of this paper with the standard second 2-descent (see e.g. Cremona [7]); I intend to address this problem at another occasion. The referee observed that mwrank gives the correct rank of the Mordell-Weil group for 19 out of the 20 open cases in Nemenzo's paper, the exceptional curve being $E_{k}$ with $k=9554=2 p l$ with $p=17$ and $l=281$; as a matter of fact, running mwrank on the 2-isogenous curve $E_{-p l}$ produces the correct rank 2 .

Acknowledgements. I thank F. R. Nemenzo for providing me with some of his unpublished results, in particular for sending me tables giving the order of Tate-Shafarevich groups of curves $E_{k}$ with rank 0 assuming BSD. I also thank the referee for his careful reading of the manuscript, and for running Cremona's program mwrank on some of the examples treated here; Table 5 was added only afterwards.

Added in proof. The latest version of John Cremona's program mwrank now chooses the 2 -isogeny that gives the minimal rank estimate. 


\section{References}

[1] N. Aoki, On the 2-Selmer groups of elliptic curves arising from the congruent number problem, Comment. Math. Univ. St. Paul. 48 (1999), 77-101.

[2] B. J. Birch and H. P. F. Swinnerton-Dyer, Notes on elliptic curves. II, J. Reine Angew. Math. 218 (1965), 79-108.

[3] R. Bölling, Die Ordnung der Schafarewitsch-Tate-Gruppe kann beliebig groß werden, Math. Nachr. 67 (1975), 157-179.

[4] N. Bruin, private communication, 2001.

[5] J. W. S. Cassels, Arithmetic on curves of genus 1. VI. The Tate-Šafarevič group can be arbitrarily large, J. Reine Angew. Math. 214/215 (1964), 65-70.

[6] -, Arithmetic on curves of genus 1. I. On a conjecture of Selmer, ibid. 202 (1959), $52-99$.

[7] J. Cremona, Higher descents on elliptic curves, preprint, 1998.

[8] G. Lejeune Dirichlet, Démonstration d'une propriété analogue à la loi de réciprocité qui existe entre deux nombres premiers quelconques, J. Reine Angew. Math. 9 (1832), 379-389; Werke I, 173-188.

[9] K. Feng, Non-congruent numbers, odd graphs and the Birch-Swinnerton-Dyer conjecture, Acta Arith. 75 (1996), 71-83.

[10] - Non-congruent number, odd graph and the BSD conjecture on $y^{2}=x^{3}-n^{2} x$, in: Singularities and Complex Geometry (Beijing, 1994), Amer. Math. Soc., 1997, 54-66; coincides with [9].

[11] A. Genocchi, Sur l'impossibilité de quelques égalités doubles, C. R. Acad. Sci. Paris 78 (1874), 423-436.

[12] T. Goto, A note on the Selmer group of the elliptic curve $y^{2}=x^{3}+D x$, Proc. Japan Acad. 77 (2001), 122-125.

[13] R. K. Guy, Unsolved Problems in Number Theory, Springer, 1981; Japan. transl. 1983; 2nd Engl. ed. 1994.

[14] H. Hasse, Bericht über neuere Untersuchungen und Probleme aus der Theorie der algebraischen Zahlkörper, Jahresber. Deutsch. Math.-Verein., Ergänzungsband 6 (1930), 204 pp., Teil II: Reziprozitätsgesetz; Reprint: Physica Verlag, Würzburg, 1965.

[15] B. Iskra, Non-congruent numbers with arbitrarily many prime factors congruent to 3 modulo 8, Proc. Japan Acad. 72 (1996), 168-169.

[16] G. Kings, Über Bedingungen für Punkte unendlicher Ordnung über $\mathbb{Q}$ auf den Kurven $E: y^{2}=x^{3}+\ell^{2} x$, Diplomarbeit Univ. Bonn, 1989.

[17] N. Koblitz, Introduction to Elliptic Curves and Modular Forms, Grad. Texts in Math. 97, 2nd ed., Springer, 1993.

[18] K. Kramer, A family of semistable elliptic curves with large Tate-Shafarevitch groups, Proc. Amer. Math. Soc. 89 (1983), 379-386.

[19] J. Lagrange, Construction d'une table de nombres congruents, Bull. Soc. Math. France, Suppl., Mem. 49-50 (1977), 125-130.

[20] - , Nombres congruents et courbes elliptiques, Sémin. Delange-Pisot-Poitou 1974/75, Fasc. 1, Exposé 16, 17 pp.

[21] F. Lemmermeyer, A note on Pépin's counterexamples to the Hasse principle for curves of genus 1, Abh. Math. Sem. Hamburg 69 (1999), 335-345.

[22] - On Tate-Shafarevich groups of some elliptic curves, in: Algebraic Number Theory and Diophantine Analysis (Graz, 1998), de Gruyter, 2000, 277-291.

[23] - Reciprocity Laws. From Euler to Eisenstein, Springer, Heidelberg, 2000. 
[24] D. Li and Y. Tian, On the Birch-Swinnerton-Dyer conjecture of elliptic curves $E_{D}: y^{2}=x^{3}-D^{2} x$, Acta Math. Sin. 16 (2000), 229-236.

[25] C.-E. Lind, Untersuchungen über die rationalen Punkte der ebenen kubischen Kurven vom Geschlecht Eins, Diss. Univ. Uppsala, 1940.

[26] O. McGuinness, The Cassels pairing in a family of elliptic curves, Ph.D. Diss., Brown Univ., 1982.

[27] F. R. Nemenzo, On the rank of the elliptic curve $y^{2}=x^{3}-2379^{2} x$, Proc. Japan Acad. 72 (1996), 206-207.

[28] —, All congruent numbers less than 40000, ibid. 74 (1998), 29-31.

[29] - e-mail message, February 4, 2002.

[30] K. Noda and H. Wada, All congruent numbers less than 10000, Proc. Japan Acad. 69 (1993), 175-178.

[31] T. Ono, On the relative Mordell-Weil rank of elliptic quartic curves, J. Math. Soc. Japan 32 (1980), 665-670.

[32] M. J. Razar, A relation between the two-component of the Tate-Safarevic group and L(1) for certain elliptic curves, Amer. J. Math. 96 (1974), 127-144.

[33] L. Rédei, Die Diophantische Gleichung $m x^{2}+n y^{2}=z^{4}$, Monatsh. Math. Phys. 48 (1939), 43-60.

[34] A. Scholz, Über die Lösbarkeit der Gleichung $t^{2}-D u^{2}=-4$, Math. Z. 39 (1934), 95-111.

[35] E. S. Selmer, A conjecture concerning rational points on cubic curves, Math. Scand. 2 (1954), 49-54.

[36] P. Serf, Congruent numbers and elliptic curves, in: Computational Number Theory (Debrecen, 1989), de Gruyter, 1991, 227-238.

[37] J. Silverman, Arithmetic of Elliptic Curves, Springer, 1986.

[38] R. J. Stroeker and J. Top, On the equation $Y^{2}=(X+p)\left(X^{2}+p^{2}\right)$, Rocky Mt. J. Math. 24 (1994), 1135-1161.

[39] H. Wada, On the rank of the elliptic curve $y^{2}=x^{3}-1513^{2} x$, Proc. Japan Acad. 72 (1996), 34-35.

[40] H. Wada and M. Taira, Computations of the rank of elliptic curve $y^{2}=x^{3}-n^{2} x$, ibid. 70 (1994), 154-157.

CSU San Marcos

333 S Twin Oaks Valley Rd.

San Marcos, CA 92096-0001, U.S.A.

E-mail: franzl@csusm.edu

Received on 28.2.2002

and in revised form on 1.10.2002 